Filo. y Lingüí. 10(2): 3-18, 1984

\title{
EL HUETAR: OBSERVACIONES SOBRE LOS MATERIALES DISPONIBLES PARA SU ESTUDIO Y SOBRE LAS HIPOTESIS EN TORNO A SUS AFINIDADES LINGUISTICAS
}

Adolfo Constenla Umaña

\section{0 . Introducción}

En los documentos costarricenses de los siglos XVI y XVII, el término huetar o güetar se emplea generalmente para referirse tanto a una lengua indígena, considerada por un documento de 1617 (Fernández, 1881-1907, V, p. 219) "la materna y general de aquella provincia", como a un grupo étnico que ocupaba, a la llegada de los conquistadores, el Valle Central y territorios aledaños a los ríos Virilla y Tárcoles (hasta la Costa del Pacífico) y al río Reventazón. Ocasionalmente, el término se usa también como nombre propio, siempre -al parecerde individuos pertenecientes al grupo étnico aludido, como en el caso del cacique Huetare (mencionado por Cereceda, véase Fernández, 1975, p. 32).

Sea cual sea el origen del término, su acepción como nombre de una lengua puede considerarse la básica, pues los huetares, en tanto hablaban una sola lengua carecían de unidad política. Formaban: "dos naciones, gobernada cada una por un príncipe o cacique supremo" (Fernández Guardia, 1975 , p. 24). La frontera entre estos dos dominios coincidía aproximadamente con el río Virilla (Fernández Guardia, ibid., p. 25): "por su situación geográfica, los dos reinos güetares pueden diferenciarse con los nombres de güetares de Oriente y güetares de Occidente. La capital del primero estaba situada en el Valle del Guarco o de Cartago...; la del segundo en el de Garabito". En vista de este hecho, el factor de identidad entre unos y otros percibido por quienes escribieron los documentos debió ser, con toda probabilidad, la lengua.

\section{Materiales disponibles de la lengua huetar}

La lengua huetar se extinguió tempranamente (muy probablemente ya en el siglo XVIII) debido al hecho de haber estado concentrados sus hablantes sobre todo en la región costarricense en que se dio la más intensa colonización española; en consecuencia, no existe la posibilidad de obtener de manera directa nuevos datos de ella. A esta limita- ción, se une otra que junto con ella nos condena, quizás de manera definitiva, a no poder aspirar a tener del huetar sino un conocimiento especialmente pobre y fragmentario: tanto los estudios sobre esta lengua como los textos escritos en ella que produjeron los misioneros de la época colonial no se han localizado hasta el momento y parecieran haberse extraviado quizás para siempre. Esto es muy desafortunado porque la producción de los misioneros, algunos de los cuales según los documentos llegaron a hablar fluidamente la lengua (se menciona en este sentido en especial a Fray Pedro de Betanzos y Fray Agustín de Ceballos), fue, al parecer, abundante (véase Lehmann, 1920, I, pp. 234-36), incluyendo gramáticas, catecismos y métodos para confesar.

¿De qué índole son, entonces, los materiales disponibles actualmente para el estudio del huetar? En las secciones siguientes me ocuparé de responder a esta pregunta.

1.1. Vocablos huetares conservados en los documentos de la época de la conquista y de la colonia

En los documentos de la época de la conquista y de la colonia aparecen una serie de vocablos que podemos considerar huetares, ya sea porque las fuentes claramente lo indiquen así (lo cual sucede sólo en una minoría de casos) o porque dicho origen se pueda inferir de las circunstancias, como en el caso de los nombres propios de personas pertenecientes al grupo étnico o de lugares situados en su territorio, que difícilmente se habrían tomado de otros idiomas.

Los vocablos en cuestión se pueden dividir en dos grupos: nombres comunes y nombres propios.

1.1.1. Los nombres comunes que hasta el momento se han encontrado en los documentos son cuatro (cf. Lehmann, ibid. pp. 236-7), tres de ellos pertenecientes a la terminología que los huetares usaban para referirse a sus dirigentes: 
ybux 'mandón', 'principal', 'hijo de cacique' taque 'principal', 'hijo de cacique'

vrivri 'hijos de caciques'

biriteca 'mujer que participaba en batallas, amazona'

La última palabra no era exclusiva de los huetares: según el documento que la menciona (Vásquez de Coronado, 1964, p. 50), así eran Ilamadas las mujeres de Coctu por "los Guetares y otras naciones".

Es interesante señalar que los elementos ybux y taque aparecen en formas reduplicadas ybuxybus y taquetaque, en contextos que indican que se trataba de secuencias con valor de plural.

El empleo de la reduplicación para pluralizar sustantivos es un pequeño detalle gramatical interesante desde el punto de vista tipológico.

Entre las lenguas chibchas de Costa Rica, al parecer sólo el guatuso emplea la reduplicación como manifestación de la categoría de plural en los sustantivos. En esta última lengua el fenómeno en cuestión está restringido a ciertos nombres de posesión inalienable, en los cuales no tiene carácter obligatorio, sino que alterna con el morfema pluralizador empleado con los sustantivos de todas las clases. Así pues, el plural de /toko:/ 'oreja' se puede expresar por medio de la forma reduplicada/toko:toko:/ o por medio de la forma /toko:mara: $\mathrm{ma} /$. El empleo de la reduplicación para expresar pluralidad se conoce también en bribri, pero en esta lengua son los adjetivos los que se reduplican cuando están referidos a un sustantivo no pluralizable (clase que coincide en general con los que designan no humanos):

と̌íči dod sadư dư

č íci dod sadư dư sadưdư 'los perros son blancos'

1.1.2. Los nombres propios, antropónimos y topónimos, constituyen la mayor parte de los términos huetares que aparecen en los documentos.

Los más abundantes son los an tropónimos, Lehmann (1920, I, pp. 228-30) recogió alrededor de 150 (algunos de los cuales, sin embargo, podrían ser topónimos, ya que a veces, en particular en los casos de nombres de caciques, es difícil determinar si se trata de nombres de individuos o de lugares).

Los siguientes son algunos ejemplos:

Abaçara, Cho, Torapo, Tiztiz, Porroçavá, Correque, Parrahara, Xoriopá, Arayuri.
Algunos ejemplos de topónimos se dan a continuación:

Aquecerrí, Puririce, Toboci, Atirro, Guicirí, Buxebus, Pacaca, Quercó, Coo, Corroce, Purapura.

Estos vocablos, a pesar de su elevado número, no pueden dar sino una contribución extremadamente modesta a nuestro conocimiento de la lengua.

Los nombres propios en cuanto tales (particularmente los an tropónimos) no tienen otro significado que su valor de términos identificadores.

Esta simplicidad extrema desde el punto de vista del contenido dificulta la identificación de posibles componentes morfológicos $y$, cuando no disponemos de otra información sobre la lengua, la hace prácticamente imposible. Así pues, bastantes nombres de persona huetares, terminan en -bá: Pacabá, Coyetebá, Taquerrabá, Curirabá, Yoçabá, pero no hay manera de saber si esa secuencia era un morfema en la lengua o no, pues lo único que estas formas tienen en común es, precisamente, el ser antropónimos. Si se trata de nombres comunes, existirían mayores posibilidades de relacionar la terminación común con algún rasgo común de significado que no fuera simplemente el de ser todos ellos nombres comunes.

Lo más lejos que podemos llegar en relación con este ejemplo es, pues, a señalar, sin ninguna seguridad, que -bá podría haber sido un formativo de temas.

Sucede en muchas lenguas que al menos algunos nombres propios estén integrados por uno o más elementos que se usan también como nombres comunes, como verbos, etc., como en castellano: Luz, Sol, Esperanza. Si esto fue así en el caso de los nombres huetares, no lo sabemos. En tanto, por ejemplo, Fernández de Oviedo (véase Lehmann, II, pp. 843-4) nos da datos sobre los nombres propios chorotegas como que el nombres del cacique de Nicoya, Nambí, significaba 'perro' y que Masaya significaba 'monte que arde', los autores de los documentos que incluyen antropónimos y topónimos huetares no parecen haberse molestado en ningún caso en darnos informaciones semejantes.

Como se puede ver, es prácticamente imposible extraer información léxica o gramatical del material que se está comentando. Del examen de los 150 términos, incluidos por Lehmann, el único detalle gramatical que se puede determinar es la pre- 
sencia de la reduplicación en unos cuantos casos, como Porroz Porroz y Tiztiz.

No sucede lo mismo en lo fonológico. A pesar de la forma muy defectuosa en que los sonidos del huetar deben haber sido representados por los que escribieron los documentos del período de la conquista y de la colonia, los vocablos que ellos nos han trasmitido nos dan a conocer muchos detalles tanto sobre lo que incluía como sobre lo que no incluía el inventario fonológico de aquella lengua.

A continuación, señalo algunas de las conclusiones que se despreden de los datos recogidos por Lehmann. Las mismas, por supuesto, son de carácter tentativo, ya que, para mayor seguridad, sería conveniente llevar a cabo una recolección exhaustiva a partir de los originales mismos de los do cumentos separando el material de acuerdo con los años en que éstos fueron escritos, con el fin de tomar en cuenta los cambios que entre el siglo XVI y el siglo XVII se dieron en la fonología y en la grafía castellanas.

Por lo que respecta a las vocales, no hay mayores indicios de que existieran distinciones fonemáticas diferentes de las que se dan en castellano. Es cierto que en algunos casos se dan vacilaciones entre $i$ y $e, u$ y $o$, pero las mismas están limitadas a sílabas átonas en que se produce el mismo tipo de fenómeno en castellano: Quircó Quercó (como diputado $\sim$ deputado), Uxarraci $\sim$ Uxarrace (como en calle $\sim$ calli, Echandi $\sim$ Echande), Corrirabá $\sim$ Currirabá (como en sepoltura sepultura). La única excepción a lo anterior es el caso de Huerra o Güirra (cacique de Turrialba), en que la $e$ y la $i$ alternan en lo que pareciera ser posición acentuada.

Ocasionalmente, se dan otras vacilaciones como en Coyetebá $\sim$ Coyutebá, Porroz Porroz $\sim$ Perroz Perroz $\sim$ Pirroz Pirroz, sobre las cuales no es posible inferir nada por tratarse de casos aislados.

Las letras usadas con valor vocálico son generalmente a, e, i, o, u. Se usan también con valor vocálico y y $v$ en posición inicial de la palabra (y de morfema en formas reduplicadas) ante consonante, representando respectivamente $i$ y $u$, como en ybuxybux y vrivri (que deben de haberse pronunciado [ibu [ibu [] y [u siu si]). Y aparece con valor vocálico a final de palabra en un caso y $v$ a principio de palabra ante vocal, en un caso también: Cachistúe $\sim$ Cachestuy; Vio.

Los símbolos empleados para escribir los sonidos consonánticos son los siguientes: (las formas citadas ejemplifican las posibilidades distributivas): a) Sonidos oclusivos sordos

p: Parrahara, Porroçavá, Piçi, Purapura, Torapo, Corripju

t: Taquerrabá, Toraci, Tiribari, Escut

$\mathrm{c}$ : (ante $a, o u$, silencio y consonante) qu (ante $e, i)$ : Cabizcara, Corroce, Queçarco, Quitao, Ibacçará, Curchac, Carecoc.

\section{b) Sonidos oclusivos o fricativos sonoros}

b/v: La $v$ aparece (con dos excepciones: Avexa y Oristuviricá) siempre ante $a$ intervocálicamente en sílaba final de palabra, posición en la que en algunos casos alterna con $b$ : Corriravá $\sim$ Corrirabá, Yoccavá Yo cabá. Estos hechos hacen muy probable que hayan representado un mismo sonido, al menos una misma entidad fonemática (quizás $[\beta])$. La $b$ se da en los demás entornos: Barva, Biltalia, Taboba, Oquiquebú, Tubucará, Tobioz, Cob.

d: Duarco, Diruamo, Dirimame. La $d$ aparece sólo en los tres vocablos anteriores. Esto contrasta con las listas de nombres de Talamanca incluidas por el mismo Lehmann (ibid. 231-4), en las cuales aparecen once formas iniciadas por $d$, a pesar de ser menos extensas. Pudiera suceder, pues, que no se hubiera dado un fonema $d$ en huetar. En vista de lo anterior, resulta especialmente interesante que dos de los nombres presuntamente huetares, Diruamo y Dirimame, procedan de Siquirres según Lehmann (ibid. $p$. 191) región que probablemente fuera de frontera entre los huetares y los talamanqueños. La baja frecuencia de $d$ podría deberse también a que en huetar existiera una alternancia alofónica entre $d$ y $r$ como en bribri o cabécar (Constenla 1981, 1982; Margery 1982)

g: Aparte de su uso en secuencia con $u$ ante vocal (equivalente a la secuencia $h u$ ), se ha observado sólo en un caso ante $g$ en una palabra que se escribe también con c: Ibagçara Ibaccará. Este sonido parece ser, pues, un alófono del mismo fonema que c/qu ( [k] ).

c) Sonido africado sordo

ch: Choboro, Chumaçara, Chiquirro, Cachistúe 
ch) Sonidos fricativos sordos

f: Aparece una sola vez en el nombre Xoriofa, que aparece transcrito otras dos como Xoriopá. Es difícil sacar una conclusión a este respecto (podría incluso tratarse de un error de copia), pero me parece interesante anotar que en una visita hecha a Quitirricí (cantón de Mora, provincia de San José), noté casos de aparente variación libre en tre la oclusiva [p] y una fricativa bilabial sorda [ $\Phi]$ en un anciano perteneciente al grupo de origen huetar que habita en aquel lugar y que actualmente sólo habla castellano.

ç/c/z/s: Entre todas estas letras se dan vacilaciones, lo cual hace suponer que pudieron representar una misma entidad fonemática: Caquerribá $\sim$ Zaquirribá $\sim$ Saquirriba, (variantes del nombre de una misma persona). Caribe $\sim$ Zaribe, Corroçe $\sim$ Corroce, Ibagçara Ibaccará. Por otra parte, ç (usada generalmente ante $a, o, u$ ) y $\mathrm{c}$ (usada generalmente ante $e, i)$, las más frecuentes y las favoritas de los documentos más antiguos, se dan sólo en posición inicial de sílaba; $z$ es inicial en algunos casos, aparece en grupos internos ante $c / q u$ y ante $p$ y es la única que aparece a final de palabra; $s$ aparece en algún caso en posición inicial de sílaba o final de palabra, y mucho más frecuente ante $p, t$ y $c / q u$.

Çabaça, Çuequin, Piçiva, Coçer, Zuquire, Zaricurá Çaricurá, Chirrez, Tobioz, Uraz, Yozpari, Yzcó, Sabucara, Ubisurrú, Aquisara, Tuxusti, Tescato, Ystaro, Patarras.

$x / j$ : El valor fonético de $x$ debe haber sido el que tenía en castellano en la época en que se comenzó a usar el alfabeto para escribir palabras huetares, [ []: Xarcopa, Xoxo, Xirober, Oxpe, Pixtoro, Axi, Pixori, Abux, Larix. La letra $j$ representó el mismo sonido en muchos lugares en el siglo XVI y en los datos se dan algunas vacilaciones entre las dos: Xima $\sim$ Jima, Caxagua Cajagua, Avexa Aveja. En un caso, Oxpe $\sim$ Ospe se ha observado vacilación entre $x$ y $s$.

d) Sonidos vibrantes

Las vibrantes simple $r$ y múltiple $r r$ son particularmente frecuentes.
Entre los primeros 82 nombres incluidos por Lehmann, 38 incluyen $r$ y 20 rr. Es difícil determinar si estos dos sonidos se oponían en huetar. En ningún caso se presentan en posición inicial de palabra y a final de sílaba sólo aparece $r$. A comienzo de sílaba interna parecen oponerse, pero hay unos pocos casos en que se dan vacilaciones, como en Procavá $\sim$ Porroçavá y Curirabá $\sim$ Currirabá, Turiarba $\sim$ Turrialba. Los siguientes son ejemplos de ambos sonidos: Chaboro, Chumaçara, Yurusti, Tiribari, Guarco, Barva, Xirober, Piror; Atarra, Churraca, Churruz, Pirritubí, Corroce.

e) Sonido lateral

La lateral / aparece en un número relativamente bajo de vocablos, tanto en posición inicial como final de sílaba, pero nunca a final de palabra: Larix, Alux, Polo, Biltalia. En un único caso se ha observado vacilación con $r:$ Turriarba $\sim$ Turrialva.

f) Semiconsonantes

gu/hu: Estas dos secuencias, entre las cuales se dan vacilaciones, representan probablemente una semiconsonante labiovelar $[w]$ que bien puede haber presentado un inicio consonantizado facultativo: Guaçara, Huerra Guirra, Parragua, Huetara, Guarco, Pirragua.

$y$ : Probablemente haya representado una semiconsonante mediopalatal sonora [j] o la fricativa correspondiente $\left[j^{\wedge}\right]$ : Yaquirre, Yurusti, Yozpari, Coyozara, Tucuyarí, Tayecara.

g) Nasales

Llama la atención la baja frecuencia de las nasales en los nombres analizados. En primer lugar, se dan solo dos: $m$ y $n$, (no hay ninguna forma con $\tilde{n})$. En segundo lugar, son contadas las palabras en que aparece cualquiera de las dos.

m: Mahan, Dirimane, Chumaçara, Cambum

$\mathrm{n}$ : Joaniqui, Guequin, In.

\section{La letra $h$}

La letra $h$, fuera de los grupos $c h$ y $h u$, se presenta en cuatro formas: Parrahara, Mahan, Cuvho y Horasca. Esta letra podría, de acuerdo con el valor que todavía tenía en la grafía castellana del siglo XVI, haber representado una aspiración, [ $h$ ]. 
La tilde que indica el acento recibe un uso bastante inconsistente, lo cual, unido a las dificultades de captación de los suprasegmentales de una lengua desconocida para la persona sin entrenamiento fonético, hace poco provechoso el especular al respecto.

En resumen, los datos procedentes de las fuentes de la época de la conquista y de la colonia sugieren que el sistema de sonidos del huetar incluía por lo menos las entidades que aparecen en los siguientes cuadros:

\section{a) Vocales}

$\begin{array}{lccc} & \text { Anterior } & \text { Central } & \text { Posterior } \\ \text { Alta } & \mathrm{i}(\mathrm{y}) & & \mathrm{u}(\mathrm{v}) \\ \text { Media } & \mathrm{e} & & \mathrm{o} \\ \text { Baja } & & \mathrm{a} & \end{array}$

b) Consonantes

Labial Dental/ Palatar Velar Laringea Alveolar

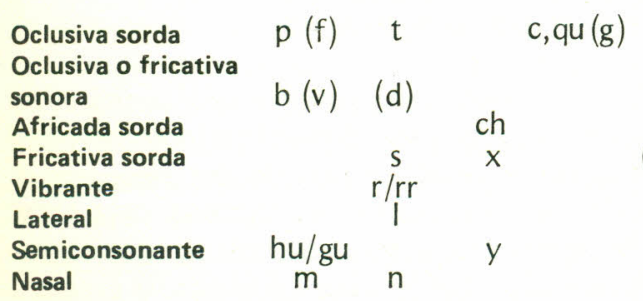

En los cuadros, las letras que tienen mayores posibilidades de estar representando un mismo fonema se han puesto en una misma casilla, clasificándolas según la articulación probable de la que se da con mayor frecuencia y poniendo la menos frecuente o más limitada desde el punto de vista distributivo entre paréntesis. Las letras que no parecen alternar con ninguna otra, pero que tienen una distribución muy limitada y una frecuencia muy baja se colocan en casillas aparte entre paréntesis. En el caso de la $r$ y $r r$ no se ha puesto ninguna entre paréntesis porque la $r r$ a pesar de tener una distribución más limitada y una frecuencia menor que la $r$, es en sí muy frecuente y pudiera haber sido una entidad fonemáticamente independiente. No obstante se han colocado las dos en una misma casilla.

Por lo que respecta a grupos consonánticos, aparentemente sólo se presenta en los datos uno tau tosilábico: $\mathrm{cr}$, en Porrocris y Cuquecre. No obstante, Escut podría incluir una secuencia inicial sc con $e$ epentética añadida al adaptarse el nombre al castellano.

Los grupos disilábicos observados más frecuentes son combinaciones de las fricativas $s \sim z$ y $x$ con las oclusivas sordas $p, t, c \sim q u$ (p.e.: $s p$, st, $s c)$, combinaciones de $r$ con $c, v o c h(r c, r v, r c h)$ y combinaciones de $c(\sim \mathrm{g})$ con $\xi, b$ y $t(\mathrm{cç}, \mathrm{cb}, \mathrm{ct}$, gç). Además se dan otras combinaciones en casos aislados como $/ t, m b, b c ̧$.

1.2. Vocablos huetares conservados como sustrato en el castellano del Valle Central

En el habla del Valle Central se dan o se han dado una serie de vocablos de los que, por ser aparentemente exclusivos de esta área (es decir, por no darse fuera del castellano de la región o de las regiones a las que en época reciente se extendió dicha variedad) y sin etimología trazable a ninguna lengua conocida que haya influido en el castellano general o en el de Costa Rica, puede sospecharse que sean elementos de sustrato huetar. Por supuesto, sólo un estudio exhaustivo que compruebe las dos características mencionadas podría darnos seguridad. Este estudio está por realizarse y en este trabajo no se intentará llevarlo a cabo, limitándose a ejemplificar los tipos de posibles huetarismos que se dan. Hay que señalar, sin embargo, que mientras no se encuentren los estudios del huetar hechos por los misioneros, esta fuente de datos es la más prometedora, pues incluye, además de nombres propios una cantidad no despreciable de nombres comunes y unas escasas palabras de otras clases que a diferencia de los nombres propios sí pueden compararse con las palabras que denotan los mismos referentes en diversas lenguas indígenas. Esto cada día se hace más factible gracias a la intensificación de la recolección de léxico, tanto del castellano del Valle Central como de las lenguas indígenas habladas en Costa Rica y en otros países del área, que se ha dado en los últimos años.

Los vocablos de aparente origen huetar se dividen en dos grupos: uno de nombres propios que incluye sólo topónimos y otro integrado sobre todo por nombres comunes y algún adjetivo.

1.2.1 Ejemplos de la serie de los topónimos (que podrían sumar varios cientos) son los siguientes:

Aquiares, Arribará, Birrís, Caraigres, Coris, Corrogres, Cuascúa, Cucubres, Chires, Chirobres, 
FILOLOGIA Y LINGUISTICA

Chures, Escazú, Guaraní, Güicasí, Irrires, Itiqu ís, Jaris, Orobres, Paires, Parrúas, Patarrá, Picagres, Pigres, Pirrís, Pocares, Porrosatí, Puruces, Purisil, Quercuá, Quirazú, Quitirricí, Siquiares, Siquirres, Surubres, Tacares, Tapantí, Taras, Tarbaca, Tatiscú, Ticufres, Tiquires, Tocorí, Toyogres, Tubures, Túfares, Tuis, Turrós, Turrubares, Turrúcares, Tusubres, Urases, Urasca, Uriche, Yacuares.

La utilidad de estos elementos como datos para el conocimiento del huetar se limita prácticamente a lo fonológico por las razones señaladas en 1.1.2. Cierto es que en este caso las precauciones deben ser ex tremadas por lo mucho que los términos en cuestión pueden haberse alterado desde la época de su adopción en el castellano hasta el presente. No obstante, la coincidencia entre estos nombres y los recogidos en los documentos coloniales en materia de sonidos, tanto por lo que respecta a los que aparecen como a los que no aparecen, es muy interesante. Nótese, por ejemplo, la Ilamativa ausencia total de consonantes nasales y de $d$. El sonido $|f|$ aparece sólo en dos nombres Túfares y Ticufres (sin embargo, ambos son de lugares más o menos cercanos a Quitirricí; véase lo dicho en 1.1.2 sobre la $f$.

En cuanto a la $g-$ si se hacen a un lado las apariciones en el grupo gu ante vocal (que representaría una [ w ] huetar) y en la secuencia $g r-$ no se da, lo mismo que en los documentos coloniales. El grupo gr no se presenta en los documentos coloniales, pero según se señaló en 1.1.2., el único grupo encontrado en ellos era $\mathrm{cr}$ (Porrocris) del cual podría descender el $g r$ actual. De igual modo, se da un grupo br que no aparece en los vocablos conservados por los documentos y que a diferencia de $g r$ no tiene en ellos un posible antepasado. Fuera de estos casos y del grupo fr que aparece en Ticufres, los nombres de posible origen huetar coinciden con los antropónimos y topónimos recogidos en la época colonial en su preferencia por el canon silábico (C) $\vee(C)$. Nótese además que en los nombres trasmitidos por ambas fuentes, existe coincidencia en el hecho de que los grupos sólo se dan en sílabas finales de palabra.

Un detalle interesante de la toponimia actual del área huetar es la cantidad enorme de nombres terminados en -res (como Tures, Túfares, Purires, etc) de los cuales resultan afines los pocos terminados en -ris (como Jaris y Coris). La difusión de estos nombres coincide muy es trechamente con los lindes del territorio que según las crónicas ocupaban los dos reinos huetares. Para comprender bien este hecho será necesario buscar en las fuentes co- loniales las formas más primitivas del mayor número posible de nombres de este tipo. A primera vista, uno podría sentirse tentado a proponer la existencia en estos vocablos de algún formativo del huetar de carácter locativo o afín, pero en estos casos se impone la mayor cautela, ya que las terminaciones comunes son a veces añadidas a posteriori por los hablantes de una nueva lengua. Así pues, por ejemplo, si el guatuso se hubiera extinguido, a partir de los nombres locales de una serie de ríos tomados de esta lengua, como Cóter, Quéquir, Tojíbar, Samen, alguien podría creer que $-n$ (usado después de otra consonante nasal) y $-r$ (usado en los demás casos) eran alomorfos de un morfema guatuso relacionado con la noción de ' $r$ ío', pero en guatuso nadie pronuncia estos nombres sino con

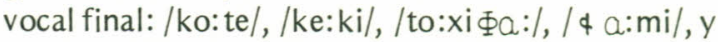
con vocal final aparecen en los datos tomados por Lehmann (op. cit.) en 1909 (y no cabe pensar que las formas castellanas locales de esos hidrónimos reflejen pronunciaciones guatusas muy antiguas porque el establecimiento de los hispanohablantes en la región es un fenómeno que turo su inicio en la segunda mitad del siglo XIX).

1.2.2. Los nombres comunes que constituyen posibles huetarismos son denominaciones de especies animales y vegetales propias del área, con la excepción de unos pocos que pertenecen a otras áreas de significado, como usú 'hueco', 'cueva', yurro 'manantial', tiqui 'calabazo grande para el agua'. El único no sustantivo que he encontrado es el adjetivo urrú.

Ejemplos de fitónimos del tipo que se están comentando son los siguientes: ascá (Styrax polyneurus Perkins), buriogre (Cordia glabra L.), cocora (Guarea microcarpa), cubá (Phaseolus coccineus L), curraré 'tipo de plátano', chirra (Tillandsia multicaulis Steud.), erepe (Calatola costaricensis Standl), guagra (Cryosophila albida Barlett), guarrazú 'tipo de pacaya', güízaro (Psidium guineense Sw.), jucó (Trema micrantha (L.) Blume), poró (Erythrina costaricensis Micheli), pu (Conostegia xalapensis (Bonpl.) D. Don.), putarrá (Smilax kunthii Killip \& Morton), quiquicirrí (Styrax Warscewiczii Perk.), quitirrí (Vernonia trifloscula HBK.), súrtuba (Geonoma edulis Wendl.), surubre (Astrocaryum confertum Wendl.), targuá (Croton costaricensis Pax), terré (Croton xalapensis HBK.), tora (Montanoa hibiscifolia Benth.), tuete (Vernonia stellaris Llave \& Lexarza), turrusí (Eugenia cartagensis Berg.), yos (Sapium sp.). 
Algunos nombres de especies zoológicas son: arragre (tipo de abeja silvestre), béquer (tipo de serpiente: Constrictor constrictor imperator), capasurí (venado que tiene los cuernos cubiertos por la piel, recogido por Gagini, en desuso actualmente), cosorró (pájaro carpintero), cuascuasa (tipo de congrejo), cusinga (tipo de tucán: Pteroglossus Frantzii), icí (en zorro icí o zorricí: Philander opossum), oropopo (tipo de búho: Pulsatrix perspicillata), quioro (tipo de tucán: Ramphastos swainsoni), pirrís (tipo de pájaro: Zonotrichia capensis), purruja (tipo de mosquito: Diptera sp.), soterré (varios pájaros de la familia troglodytidae), yárreco (tipo de cangrejo), yerre (tipo de gallina de monte: Crypturellus cinnamomeus), yigüirro (tipo de tordo: Grayi), yuré (tipo de paloma: Leptotila verreauxi).

\subsection{Huetarismos conservados en el cabécar norteño}

De mis propias observaciones y del trabajo de Margery (comunicación personal) sobre el cabécar, se desprende que esta lengua presenta una división en dos dialectos principales: el hablado en el Valle de la Estrella y en Chirripó, que denomino cabécar norteño, por una parte, y el hablado en el Valle de Talamanca y en la vertiente del Pacífico, que denomino cabécar sureño.

El cabécar norteño estuvo en la época precolombina en contacto con el huetar en la región correspondiente al actual cantón de Turrialba, de modo que es de esperarse que ambas lenguas hayan intercambiado elementos.

Parece, incluso, que lo más probable haya sido que la influencia mayor se diera del huetar al cabécar, por el carácter de lengua general o franca que el primero tenía de acuerdo con las fuentes coloniales, según se tratará más adelante.

Reconocidas las posibilidades señaladas en el párrafo anterior, cabe preguntarse cómo se pueden reconocer los posibles huetarismos en el cabécar norteño. La respuesta reside en los resultados de la aplicación del método comparativo de la lingüística diacrónica. El paso fundamental de este método es el establecimiento de matrices de correspondencias entre los sistemas fonológicos de las lenguas comparadas en las que estos se relacionen metódicamente como conjuntos y elemento por elemento. Como lo señala McQuown (1954, pp. 502-3):

"Estas matrices se establecen por medio de la búsqueda de un conjunto de correspondencias, sonido por sonido, en el material léxico de una pareja de lenguas. Las correspondencias buscadas deben ser regulares, esto es, en un entorno fonológico dado, un sonido particular de una lengua debe tener siempre la misma correspondencia en la segunda lengua..."

Una vez establecida una matriz del tipo descrito, la misma sirve para determinar si una pareja de formas de dos lenguas descienden de un mismo étimo, es decir, de una misma forma de la proto-lengua. Si dos formas de distintas lenguas cada una cumplen con lo previsto por la matriz, ambas son formas heredadas directamente. Si de las dos formas hay alguna que no cumple, esto indica que se ha producido un cambio de tipo distinto al regular, es decir al cambio fonológico. Uno de los tipos de cambio no fonológico es el préstamo, es decir, la adopción de una forma de otra lengua, esté ésta poco distanciada o muy distanciada de la que toma en préstamo un elemento suyo. Como señala McQuown (ibid., p. 510):

"Empleando la matriz de correspondencias fonéticas se separan las ovejas heredades de las cabras tomadas en préstamo y se hace posible trazar la historia lingü fstica".

En el caso que nos ocupa, las matrices de correspondencias del cabécar con toda una serie de lenguas de la familia chibcha se han establecido (Constenla, 1981), y en la actualidad está en preparación un diccionario pandialectal cabécar (proyecto a cargo de E. Margery de la Universidad de Costa Rica), de modo que se comienza a estar en la posibilidad de reconocer los préstamos que dicha lengua haya recibido, tarea que hace pocos años era impensable y que hoy, aunque factible, está por realizar.

Por el momento, estoy en condicioes de dar un único ejemplo. Los tucancillos verdes (Aulacorhynchus) se denominan en guatuso /taka: raka:ra/ y en bribri /dakałàk!. Las raíces de ambas formas /taka:ra-/ y /dakay $a^{2}-1$ presentan correspondencias perfectamente regulares (compárese, por ejemplo, guatuso /tu a:/, bribri /dauá/ 'tabaco'; guatuso/puru:puru:/ bribri /bữũ (wak/ 'purruja') que llevan a la reconstrucción de un étimo/*dək ərə/.En cabécar, como en bribri, a la $/ \mathrm{t} / \mathrm{y} / \mathrm{l} / \mathrm{r} / \mathrm{guatusas}$ corresponden respectivamente $/ d /$ y $/ \mathbb{T} /$, sin embargo, examinando los ficheros de E. Margery, he encontrado la forma del cabécar norteño/taka sák/'tucanciIlo orejinegro' (Selenidera spectabilis), obviamente relacionada, pero doblemente irregular desde el punto de vista de las correspondencias fonéticas. 
Ahora bien, esta forma no procede del bribri ni mucho menos del castellano que son las únicas lenguas fuera del huetar que han estado en contacto con el cabécar norteño. La conclusión más aceptable es, pues, que el término es un huetarismo.

2.Posición del huetar en las clasificaciones de las lenguas del área

Brinton (1897) clasificó el huetar como miembro de un grupo lingüístico talamanqueño, considerando que estaba especialmente relacionado con el cabécar.

Cyrus Thomas (1902) lo incluyó entre las lenguas de Centroamérica pertenecientes a la familia chibcha sin relacionarlo especialmente con ninguna otra.

Rivet (1912) lo colocó en su rama talamanca barbacoa de la familia chibcha junto con el guatuso, el cuna, el boruca, el cabécar, el térraba, el bribri, el chirripó (cabécar) y las lenguas barbacoas.

Lehmann (1920) consideró que constituía un subgrupo por sí solo dentro del grupo talamanqueño en el que situó las lenguas del sur de Costa Rica (bribri, cabécar, térraba y boruca).

A partir de estas clasificaciones, la mayor parte de las que se han hecho posteriormente han considerado el huetar como miembro de un grupo talamanqueño de la rama occidental (o talamanca barbacoa) de la familia chibcha perteneciente a su vez al filo macrochibcha (jijón y Caamaño, 1943; Mason, 1950; McQuown, 1954; Tovar, 1961; Voegelin, 1965).

Al lado de esta posición más extendida se da la de Schmidt (1926) quien agrupó al huetar y otras presuntas lenguas extintas (voto, corobicí, suerre, quepo) junto con el guatuso y el rama dentro de una de las divisiones de la rama noroccidental (o talamanca - dorasque) de la familia chibcha.

Loukotka $(1944,1968)$ basó su clasificación en la de Schmidt por lo que atañe a las relaciones más estrechas del huetar, que situó en uno de los grupos en que de manera directa divide la familia, el grupo guatuso, con las lenguas antes mencionadas excepto el rama.

Otras clasificaciones como las de Swadesh (1959) y Greenberg (1960) no incluyen el huetar.

Fuera de Brinton y de Lehmann, ninguno de los autores mencionados llegó a dar argumentos en favor de su clasificación del huetar. En las siguientes secciones me ocuparé de demostrar que los argumentos de Brinton y de Lehmann, lo mismo que los dados en favor de diversas hipótesis por otros autores que no produjeron clasificaciones, carecen de validez científica y en consecuencia no pueden tomarse en cuenta siquiera como indicios en favor de las relaciones propuestas.

\section{Hipótesis sobre las afinidades lingüísticas de los huetares}

Por lo escaso de los materiales lingü ísticos disponibles, desde que se comenzó a especular sobre las afinidades de los huetares se comprendió que la tarea era una de particular dificultad. Así pues, Peralta (1893) afirmó: "es casi imposible determinar las afinidades étnicas de los Güetares mientras no se descubran en alguna biblioteca los vocabularios que debieron escribir franciscanos tan hábiles en el arte de sus lenguas como Fray Pedro de Betanzos, Fray Juan Baptista y otros que fundaron varios conventos y catequizaron a los Güetares de Cartago" y Gagini (1917) consideró que el problema era el "punto negro" de la etnografía costarricense.

\subsection{Hipótesis de Brinton}

No obstante, Brinton (1897) creyó haber hallado la solución en un léxico de 123 rubros, conocido como Vocabulario de los indios de San José de Costa Rica y recogido a mediados del siglo XIX, que él había adquirido como parte de la colección de manuscritos Berendt. Según notó acertadamente Brinton, la mayor parte de las palabras incluidas en dicho documento eran muy semejantes a las palabras correspondientes de las lenguas talamanqueñas (de hecho, a la luz de nuestros conocimientos actuales, se ve claramente que la lengua del vocabulario es el cabécar). En vista de que el vocabulario había sido recogido en las "cercanías" de San José, esto es, en el territorio señalado por las fuentes históricas como parte del dominio huetar, Brinton concluyó que el vocabulario era una muestra de la lengua huetar y que con esto quedaba demostrado el estrecho parentesco existente entre el huetar y las lenguas cabécar y bribri. Brinton expuso otros argumentos de menor peso en favor de su hipótesis: que las 5 palabras recogidas por Benzoni en Suerre en 1544 se parecían a las correspondientes de las lenguas talamanqueñas, que varios nombres geográficos de la Meseta Central (Escazú, Tarrazú, Irazú, etc.) incluían un sufijo $-z u$ que en 
cabécar significaba 'cerro' y que una palabra huetar recogida por Juan Vásquez de Coronado, biriteca 'amazona' tenía correspondencia en las lenguas talamanqueñas.

El argumento principal de Brinton fue objetado primeramente por Thomas y Swanton (1911, p. 85-6) quienes refiriéndose al vocabulario de San José señalaron:

\begin{abstract}
"este se obtuvo hace aproximadamente 44 años de algunos nativos que resid f́an cerca de San José, pero pareciera que no se dijo una sola palabra acerca de su relación con la tribu güetar. Los indicios reales en consecuencia, se limitan al hecho de que el vocabulario se obtuvo de indios que vivfan en la región anteriormente, comprendida en el territorio gůetar. Se considera más seguro, ..., incluir por ahora el idioma en el grupo talamanqueño".
\end{abstract}

Sin embargo, fue Gagini (1917, p. 56) quien lo rebatió totalmente habiendo determinado que el Vocabulario de San José "fue hecho por el señor Riotte en Orosi o Tucurrique" y que "estos lugares estaban desde el siglo XVIII habitados, no por Huetares, sino por Talamancas".

Por lo que atañe a los otros argumentos de Brinton, hay que anotar lo siguiente:

a) Cabe la posibilidad de que al menos dos de las palabras recogidas en suerre tengan relación con palabras cabécares (Constenla, 1981, p. 53), pero el mismo Brinton $(1897$, p. 497$)$ reconoció que:

"el "suerre" aunque Berendt y otros lo hayan considerado idéntico al güetar, no se sabe positivamente que lo haya sido $y$, geográficamente, parece haberse hablado en la costa norte, a lo largo del río del mismo nombre, a alguna distancia de la provincia de los güetares históricos".

b) En cuanto al presunto sufijo $-z u$, que según Brinton estaría contenido en topónimos como Irazú y Escazú, hay que señalar, por una parte, que en cabécar existe, en efecto, un elemento /- $\phi_{\sigma} /$ que se presenta combinado con / $\mathrm{k} \tilde{a} /$ ' lugar, tiempo' en la palabra / $k \tilde{a} \hat{c}_{\infty} /$ 'cerro', pero también es cierto que este elemento no se presenta, aparentemente, fuera de dicha palabra, ni en topónimos ni en ningún otro tipo de vocablo. Por otra parte, no hay ninguna razón para suponer que el elemento cabécar en cuestión estuviera comprendido en nombres como Tarrazú; Irazú y Escazú, careciendo como carecemos de toda base científica para tratar de establecer la etimología de los mismos. Desafortunadamente el estudio de la toponomia del área, no sólo en este casi sino que en la inmensa mayoría de los trabajos que se han ocupado de ella, se ha llevado a cabo de manera caprichosa y ametódica, sin tomar en cuenta los principios básicos de la investigación toponímica seria (como se exponen, por ejemplo, en Corominas, 1972, pp. 128-156).

c) Finalmente, no se pueden tomar en serio las especulaciones de Brinton sobre la etimología de biriteca que consideró compuesta por una palabra era que no identificó (probablemente el cabécar / alákl $\mathrm{Q} /$ o por la palabra térraba wa-re (/ywal //'mujer') más el verbo bribri ituk (/tôk/) 'golpear, derribar de un disparo'). Las mismas van en contra de los principios más elementales de la investigación etimológica (como se exponen, por ejemplo, en Szemerény, 1962). Además, como lo hizo notar Gagini (1917, p. 56) la fuente colonial (Vásquez de Coronado, 1964) señala que el nombre biriteca era empleado por "los güetares y otras naciones".

\subsection{Hipótesis de Gagini}

Como se habrá notado, algunas de las críticas mås acertadas a la posición de Brinton provinieron de Gagini. Este último autor, sin embargo, rechazó la hipótesis de Brinton únicamente para proponer otra abiertamente descabellada (Gagini, ibid. p. 62): que el idioma huetar había sido "una mezcla del tarasco" (lengua aislada hablada en el estado de Michoacán, Méjico, véase Campbell, 1979) "con lenguas del grupo utoazteca... y aún es posible que entre ellos hubiese tribus chorotegas, como se verá más adelante, y aún caribes".

La obsesión tarasca le vino a Gagini de la manera arbitraria en que trató de resolver el problema de las afinidades del huetar: tomó la palabra ybux o ebux 'principal' antes mencionada y se dedicó a buscar conexiones etimológicas en vocabularios de toda clase de lenguas americanas. Finalmente encontró que cabezà en tarasco se decía ehpu y eso le bastó para creer que había encontrado la solución.

La exposición de Gagini (ibid., pp. 63-70) es una larga sucesión de identificaciones arbitrarias en que a los pocos nombres comunes y a los numerosos gentilicios y topónimos del área huetar se les asigna "etimologías" cuya única base es el parecido que él creyó ver entre ellas y cualquier voz contenida en cualquier léxico del tarasco o de la 
amplia gama de lenguas que pensaba se habían mezclado para constituir el huetar.

El siguiente es un ejemplo de las mismas (ibid., pp. 64-5): "Abacara y Chucasque parecen aztecas; Cobobici, Cahita; Cobux, es el tarasco copux ciruelas; Xoquia el azteca xoquia, plural de xoquitl, barrizal; Cobux Ciruelas y Xoquia, Barrial, dieron después Cubujuquí, nombre antiguo de Heredia. Yurusti... es Yolotzin, "corazoncito"; y por último Barva... puede ser el azteca Palapan, "en el río negro u oscuro", nombre que de acuerdo con la fonética huetar debía dar Baraba ... que pronunciado como esdrújulo, según hacen los Tarahúmaras y otros pueblos, dió Barba".

\subsection{Hipótesis de Fernández Guardia y Pérez Zeledón}

Con posterioridad a Gagini, Ricardo Fernández Guardia (Pérez Zeledón, 1918) renovó la tesis de la identidad del huetar y el "viceita, talamanca o bribri", basándose en el hecho de que en la proposición hecha al rey en 1617 por el capitán Diego

- del Cubillo (Fernández, 1881-1907, V, pp. 218-22) se dice lo siguiente: "Así mismo me obligó a que ... pondré el catecismo y doctrina cristiana en la lengua materna y general de aquella provincia que Ilaman güetar, de manera que con facilidad los religiosos puedan administrar los sacramentos y los indios entender la fee y se puedan confesar". Según Fernández Guardia el capitán del Cubillo planteaba esto como un medio para lograr la conquista de Talamanca, pareciendo pues lógico que la lengua hablada en dicha región fuera el huetar.

La posición de Fernández Guardia fue adoptada también por Pedro Pérez Zeledón (1918) (quien ante la objeción planteada por Gagini de que no había razón alguna que impidiera que en un país dominara un habla como lengua general $y$, al propio tiempo, en secciones dadas de ese país, se hablaran dialectoslocales)argumentó que si lo que se pretendía concretamente era conquistar Talamanca y la lengua nativa de dicha región no era el huetar sino otra, carecería totalmente de sentido que el capitán del Cubillo se hubiera comprometido a hacer que se tradujeran los textos religiosos al huetar, por más que este fuera la lengua general de Costa Rica, pues lo que se necesitaba para facilitar aquel objetivo era que se pudiera evangelizar en la lengua vernácula. Pérez Zeledón argumentó también que dado que a) fray Agustín de Ceballos en una carta al rey del 10 de marzo de 1610 afirmaba conocer tres lenguas indígenas de la provincia (religiosa) de Nicaragua y Costa Rica; b) que en un documento de 1608 (Informaciones de méritos y servicios de los frailes menores de San Francisco, Fernández, 1881-1907, VIII, p. 47-101) se señala que las lenguas habladas por fray Agustín eran la de Nicaragua (nicarao, es decir nahoa), la de Nicoya (chorotega) y huetar, y c) que era "bien sabido que fray Agustín" había "trabajado asiduamente por largos años en la evangelización de Talamanca" y hab ía predicado a los indios de dicha región "en su lengua nativa, a más de haber compuesto en güetar catecismo, confesionarios y doctrina", se imponía la conclusión de que no pudiendo haber sido ni el chorotega ni el nahoa las lenguas que usara "tuvo que echar mano del güetar que era la otra única lengua de naturales que entendía y hablaba".

Además de estas razones, aparentemente bien fundamentadas, Pérez Zeledón añadió otras de pretenciones lingü ísticas cuya fal ta de valor es obvia a primera vista por tratar de establecer parentesco lingü ístico sobre la base de presuntas coincidencias entre segmentos del plano de la expresión del huetar y de la "lengua de Talamanca" sin aten der para nada al plano del contenido, en contradicción con los principios más básicos de la lingüística diacrónica:

'fácil es la tarea de perfeccionar la prueba de la tesis en discusion, mediante el estudio comparativo de la estructura de las palabras pertenecientes al dialecto vivo de Talamanca, de una parte, y de las voces que nos quedan de la lengua güetar, por la otra ... Esos nombres de personas o cosas güetares pasan de 500 vocablos, y sus raf́ces, terminaciones y demás elementos constituyentes proclaman a mi juicio la identidad de origen de uno y otro grupo de palabras.

No es este lugar adecuado para entrar en semejante afån; y de paso diré, sin embargo, que las terminaciones en abi y abí, aba y abá, aca y acá, ura y urá, ibi e ib f, ari y arr f, irri e irrf́, asi y asf́, ica e icá, eva y evá, aru y arú; asf como las rafces: tari, turi, tiri y tara; curi y cari, saqui y uqui, uru y ura; arra, acha, buca y guaca; chuma y chura, taye, cabi, queso, tabi, tubú, tocu, guti, poco oro, etcétera, son tan frecuentes paralelas en la lengua de Talamanca y en las reliquias de la huetar que han llegado a nosotros, que no pueden (sic) ponerse en duda su inmediato parentesco, por no decir manifiesta identidad".

A continuación paso a refutar la argumentación de Fernández Guardia y Pérez Zeledón que se acaba de presentar.

Por lo que atañe al argumento relacionado con la proposición del capitán del Cubillo cabe señalar que, cuando los documentos coloniales califican a una lengua como general,, éste término no es empleado en ellos de manera imprecisa, sino con toda 
exactitud en el sentido que actualmente le dan algunos lingüístas. El término en cuestión es sinónimo del quizás más frecuente lingua franca, que Lázaro Carreter (1962) define como: "lengua o variedad dialectal única que adoptan para comunicarse individuos de lenguas o dialectos diferentes cuando han de mantener un contacto frecuente". A este respecto señala Tovar (1961): "lengua general es aquella reconocida en calidad de tal por los conquistadores, pero aceptando un hecho anterior a la conquista misma. Son por lo mismo lenguas generales el Nahuatl de los Aztecas de Méjico y el Quechua de los Incas del Perú".

Existen motivos para que el huetar en efecto hubiera servico en la época anterior a la conquista como lengua general. Por una parte, su distribución territorial hacía que estuviera en contacto con varios de los otros grupos indígenas de lo que actualmente es Costa Rica y, por otra, de acuerdo con los informes de la época de la conquista, constituían un grupo especialmente poderoso desde el punto de vista político y militar que, por ejemplo amenazaba a los chorotegas (véase Fernández, 1975 , p. 16) y tenía a los votos reducidos a condición de tributarios (véase Fernández Guardia, 1975, p. 109).

Ahora bien, los misioneros españoles, cuando existía una lengua general, con frecuencia no se preocupaban por las lenguas de carácter más local, como señala Tovar (1961, p. 189): "en muchos lugares los misioneros adoptaron simplemente la lengua general y la ex tendieron entre los indígenas de lenguas menores". En el caso de los conquistadores, esta actitud era todavía más decidida (Tovar, ibid.): "la organización colonizadora de la Corona desconoció las lenguas menores". Como señala este mismo autor (ibid., p. 190) la lengua general era la "llave maestra en el complejo mundo indígena".

Este hecho ocasionó que, en algunos casos incluso, los españoles fueran responsables de la difusión e imposición de una lengua general por territorios en los que anteriormente no se había hablado o era muy poco conocida, como fue el caso de la penetración del quechua en el norte de Argentina de acuerdo con Morínigo (1959, pp. 80-98).

En las palabras de Tovar (1961, p. 189): "la convivencia de lenguas que surge a partir de los últimos años del siglo XVI ... determina las relaciones entre el español y las lenguas indígenas. Su fórmula podría ser: utilización de la lengua invasora por indígenas, utilización de las grandes lenguas generales por conquistadores y misioneros y tam- bién por ind igenas hablantes de lenguas menores". Como se puede ver, fue su conocimiento defectuoso de las circunstancias linguísticas del período de la conquista y de la colonia lo que llevó a que Fernández Guardia y Pérez Zeledón creyeran que el hecho de que el capitán del Cubillo hablara de poner el catecismo y la doctrina cristiana en huetar, esto tenía que significar que aquellos a los que se iba a catequizar serían personas cuya lengua materna fuera el huetar. Pero por otra parte, sus conclusiones erradas se debieron también a una lectura descuidada del documento en cuestión, porque el propósito de del Cubillo era, como lo dijo muy claramente, reducir y pacificar no solo a los indios de talamanca sino que también a (Fernández, 1881-1907, V, p. 218) "los demás que están por rreduzir en la dicha provincia" (Costa Rica): En su proposición, por ejemplo, del Cubillo menciona en este sentido varias veces a los votos

En cuanto al segundo argumento de Pérez Zeledón se puede señalar en primer lugar que, de acuerdo con lo que se acaba de exponer, aunque fuera cierto (como en efecto parece) que Fray Agustín sólo hablara náhuatl, chorotega y huetar y probablemente hubiera empleado esta última lengua en sus prédicas en Talamanca, esto no quiere decir en modo alguno que los indios talamanqueños tuvieran el huetar como su lengua materna. Pero por otra parte hay otros factores que es necesario tomar en cuenta. La presencia de los españoles en Talamanca durante el siglo XVI fue bastante esporádica y no fue sino a principios del XVII en que, con la fundación de la ciudad de Santiago de Talamanca (1605), esta presencia se hizo más permanente, de modo que, cuando se escribieron las Informaciones de méritos y servicios de los frayles menores de San Francisco en 1607 y 1608, el conocimiento que se tenía de la región no podía ser muy profundo. Además, de las mismas informaciones se desprende que el misionero a cargo de ella fue Fray Juan de Ortega. Todo parece indicar que la participación de Fray Agustín de Ceballos, al menos durante aquellos años, en las misiones de Talamanca debe haberse limitado a alguna visita en su condición de provincial de Costa Rica. La superficialidad del conocimiento que podía tener Fray Agustín de Ceballos de la situación lingü ística de Talamanca se hace obvia al leer el memorial que le envió al Rey en 1610, en el cual aunque da algunos datos muy interesantes desde el punto de vista etnográfico no menciona en ningún momento una sola de las lenguas habladas allí. Este conocimiento 
superficial no era exclusivo de él. En las Informaciones de méritos tampoco aparece mención específica de las lenguas talamanqueñas, lo cual contrasta profundamente con la exactitud extraordinaria con que, 100 años más tarde, Fray Antonio de Andrade y Fray Pablo de Rebullida delimitan los dominios de las lenguas cabécar, bribri y tiribí (Fernández, 1881-1907, V). No es de extrañar este hecho, ya que de acuerdo con Fernández Guardia (1975, p. 189) no fue sino a partir de 1689 que "entraron en su apogeo las misiones de Talamanca".

Por otra parte, para la época del Memorial de Fray Agustín de Ceballos, a apenas medio siglo del establecimiento de los españoles en su territorio, el güetar mantenía su vitalidad y su vigencia como lengua general, sintiendo los misioneros poca necesidad de dominar las lenguas más locales. Pero un siglo más tarde, destruida la estructura precolombina de relaciones entre el centro del país y las otras regiones, diezmada y cada vez más absorbida la población huetar, el aprendizaje de su lengua por individuos de otros grupos habría perdido vigencia por completo, de modo que los misioneros se verían en la necesidad absoluta de aprender las lenguas de Talamanca para poder predicar en aquella región.

\subsection{Hipótesis de Walter Lehmann}

Lehmann (1920, I, p. 179) era de la opinión de que no debía de estar muy separada de las actuales lenguas talamanqueñas. Sus argumentos en favor de esta idea no son, sin embargo, mejores que los de sus predecedores, ya que se basan en la identificación de partes, arbitrariamente segmentadas de topónimos y antropónimos huetares con morfemas de las lenguas talamanqueñas. Así pues, considera que en Accerri (una de las formas del actual topónimo Aserrí en los documentos de la época de la conquista y comienzos de la colonia) la primera sílaba ac significa piedra y por lo tanto coincide con el bribri / $\hat{a} k$ / 'piedra', que en Cobarrá y Peara hay un elemento ara arra 'hijo, niño' que coincide con el bribri / alá/ 'hijo niño', que en Choboro aparece un boro que corresponde al bribri/buł ǔ ?/, que en Turrúcares está contenido un car que significa árbol como el bribri/kál/, que en Tiribí está comprendido de ti 'río' emparentado con el bribri /di?/ 'agua, río', etc., etc. En ningún momento aclara, sin embargo, con cuál fundamento hace estas identificaciones, ya que ninguna fuente documental nos da el significado de los topónimos y los antropónimos citados, si es que tuvieron otro que el de designar a las personas y los lugares en cuestión.

\subsection{Especulaciones posteriores}

Después de Lehmann, el tema de la identidad lingü ística de los huetares pareciera no haber recibido mucha atención en ningún estudio, sino tan solo haber sido tocado con ligereza en obras de carácter eminentemente especulativo y acientífico mo (para citar sólo el ejemplo más reciente) la de Bâkit (1981) en la que se desarrolla una fantasía, esta vez de vinculación sudamericana, basada en el "descubrimiento", por parte del autor, de "la etimología" de varios nombres nombres de origen huetar iniciados por $g$ (como güetar, Garabito, Guarco, etc.), que en su opinión se derivarían de la palabra guaraní guarini 'guerra'.

\subsection{Conclusiones sobre las hipótesis analizadas}

De lo expuesto queda claro que hasta el momento no se han dado argumentos aceptables desde el punto de vista lingüístico para determinar las afinidades lingü ísticas del huetar y que, en consecuencia, no se ha demostrado su pertenencia a ninguna familia lingü ística, mucho menos su inclusión en un grupo o incluso subgrupo de una familia.

\section{Argumentos en favor de la inclusión del huetar en la familia chibcha}

Existen grandes diferencias entre las posibilidades de confirmación de las distintas hipótesis tratadas en 3. La mejor orientada de ellas, por razones obvias de localización geográfica, es la que asigna la lengua a la familia chibcha. Por medio de la aplicación del método comparativo a los elementos que aparentemente no pueden ser sino huetarismos conservados como sustrato en el castellano del $\mathrm{Va}$ Ile Central he obtenido resultados que la apoyan: al menos ocho de dichos vocablos proceden de étimos que se dan en otras lenguas indígenas costarricenses de origen chibcha (para mayor información sobre las correspondencias fonéticas y las formas reconstruidas véase Constenla, 1981):

burú 'tipo de abeja silvestre'. Bribri /bứluà k/ 'abeja'.

capasurí 'venado que tiene los cuernos recubier-

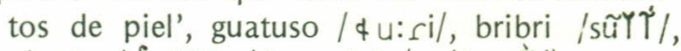
térraba /. u'Y ì / 'venado'. $(</ *$ surî̀/). 
No he observado en la actualidad el uso de este término recogido por Gagini en su Diccionario de costarriqueñismos. El que esta fuente no indique el lugar donde se usaba la palabra, hace casi seguro que ésta fuera propia del habla del Valle Central, que es sin duda su objeto principal de descripción. Gagini tiende a dar indicaciones sobre el área de procedencia de los vocablos casi únicamente cuando no es el Valle Central (véanse, por ejemplo, los artículos poró y elequeme).

cosorró 'pájaro carpintero', guatuso/ \& oro: ro/, boruca/kuxs rot/ ( $</ *$ soro $-/$.

La raíz en guatuso es / $\$$ or o:/, /-ro/ es un formativo consistente en la reduplicación de la última sílaba de la raíz, la / $\$$ / guatusa es correspondida por /s/ en las otras lenguas chibchas de Costa Rica excepto en térraba, en que aparece $/ \int /$.

En boruca, el grupo $/ \times s \varsigma /$ es señal de la existencia de un linde morfemático previo (kux/ y / srot/ (aunque esta palabra parezca ser inanalizable sincrónicamente): el grupo / ss / fuera de este caso sólo se ha observado como inicial de morfema.

cusinga 'tipo de tucán' (Pteroglossus torquatus, Pteroglossus Frantzii), guatuso

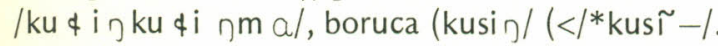

En guatuso $/-\mathrm{ma} / \mathrm{y} / \mathrm{d}$ son formativos de temas. La / g/ final del boruca proviene de la nasalidad vocálica de la protolengua, la / g/ guatusa tiene un origen completamente diferente.

poró 'tipo de árbol' (Erythrina costaricensis Micheli), guatuso /po:lo //, bribri /baYó/, cabécar /bayo/ (</*borò/).

purruja 'tipo de mosquito' (Diptera sp.), guatuso /puru:puru:/, bribrị /bữ̛̛̃ โuak/, cabécar /bũlứ suák/(</*burử?/).

En guatuso aparece un formativo de temas consistente en la reduplicación total de la raíz: en bribri y cabécar $/-\lceil/$ y $/-s /$ son también formativos de temas y /-uak/ aparece en nombres de insectos que suelen darse en grandes grupos. El huetar probablemente presentaba una forma con un formativo / - $/$ / como el bribri: /*puru [/ al cual se le habría añadido la vocal $(-a /$ 'femenino' en castellano constituyendo un $/ *$ puru $\int a /$ que por el cambio de $/ \int /$ |x/ que tuvo lugar en el siglo XVII, habría dado el actual /puruxa/.

urás 'hijo menor', guatuso (usary 'hijo varón'. $(</ * u$ rə-l).

Esta palabra recogida por Gagini, aparentemente ha caído en desuso. La /-s/ final probablemente fuera un formativo de temas como la $1-\eta /$ guatusa.

yurro 'manantial, vertiente', bribri /jół/ 'chorro, corriente de agua, cascada' (probablemente $\left.</ *_{\odot} \odot-/\right)$.

Fuera de estos casos que parecen etimologías seguras, hay otros en que existe alguna posibilidad, como arrangre 'tipo de abeja pequeña negra' que podría estar relacionado con el térraba /o $5-1$ (cf/o rdio/ 'miel', /dio/ 'líquido') o urrú 'apolillado, carcomido (referido a madera)' que, como ha señalado Agüero (1962, p. 209) podría tener el mismo étimo que el bribri /uYú/, cabécar /hưù/ 'balsa', embarcación que se hace de la madera del mismo nombre (en castellano), que es extremadamente suave.

Las formas del sustrato comentadas en los párrafós anteriores aclaran uno de los puntos dudosos que se plantearon en 1.1. en relación con el sistema fonológico del huetar: aparentemente en esta lengua $[r]$ y $[r]$ se oponían. El fonema $|s|$ era reflejo de dos protofonemas, $|* s|$ y $|*| \mid$, en tanto que $|r|$ era reflejo de uno solo $/ *_{r} /$.

\section{Dificultades de la subclasificación del huetar}

Como se señaló antes, la mayor parte de los autores de clasificaciones de las lenguas chibchas, lo mismo que los que en el pasado formularon hipótesis sobre las afinidades del huetar, subagruparon esta lengua con otras determinadas. La facilidad con que esto se creyó factible regleja más que nada la falta de conocimiento que hasta hace poco ha existido sobre la profundidad de la separación que existe entre las lenguas chibchas. La observación del cuadro que se incluye de porcentajes de términos compartidos en una lista de 126 rubros de vocabulario básico (incluidos entre los que integran la lista glotocronológica de 215 rubros, véase Swadesh, 1955) que Ilené para 13 lenguas de la familia chibcha permite ver que fuera del caso del bribri y del cabécar (que comparten un $71 \%$ ), la relación léxica, incluso en el 
caso de lenguas vecinas, es bien distante (un 23\% entre el bribri y el térraba; un $20 \%$ entre el guatuso y el rama, para citar dos casos).

Tomando en cuenta la realidad reflejada por el cuadro $y$ el hecho de que no son muchos los elementos de sustrato huetar presentes en el castellano de Costa Rica que han resultado tener términos emparentados en las otras lenguas de la familia habladas todavía en el mismo país, no es arriesgado pensar que las relaciones entre esta lengua y las otras de la familia bien pueden haber sido tan distantes como las que de acuerdo con el cuadro se dan en el caso de, por ejemplo, el guatuso. Esto no quiere decir que no deba intentarse sacar conclusiones sobre la mayor o menor afinidad del huetar con otras lenguas de la familia a la que parece haber pertenecido. Eso sí, es un claro indicio de que no se puede ser muy optimista y de que la cautela debe ser extremada, pues la subclasificación con frecuencia es asunto difícil, aún cuando los datos disponibles sean relativamente abundan- tes, debido a los efectos de fenómenos como los cambios coincidentes aunque independientes (originados en tendencias presentes en el sistema de la lengua madre) y el contacto entre lenguas. Lo escaso de los términos de aparente origen huetar para los cuales se han encontrado etimologías chibchas, hace por el momento imposible apreciar el verdadero carácter de las innovaciones de esta lengua ( $y$, como es bien sabido, las innovaciones son el criterio por excelencia en materia de subagrupación de lenguas emparentadas). Así pues, formas como purruja, poró, / taka rák/ hacen pensar que el huetar pudo haber llevado a cabo el mismo tipo de fusión de las oclusivas sonoras con las correspondientes sordas que hizo el guatuso (Constenla, 1981), sin embargo, no se puede estar seguro, porque no se sabe qué sucedió en huetar con las oclusivas sordas $(/ * \mathrm{t} /$ y $/ * \mathrm{p} /)$ de la protolengua, pues no se han encontrado formas con étimos que las presenten $y$, además, hay un caso, burú, en el que $/ * \mathrm{~b} />b$.

Porcentajes de vocablos compartidos por 13 lenguas chibchas en una lista de 126 rubros

paya rama guatuso bribri cabécar térraba boruca move bocotá cuna cágaba bintucua

\begin{tabular}{|c|c|c|c|c|c|c|c|c|c|c|c|}
\hline $\mathrm{Pa}$ & & & & & & & & & & & \\
\hline $\mathrm{Ra}$ & 0,15 & & & & & & & & & & \\
\hline Guat. & 0,12 & 0,20 & & & & & & & & & \\
\hline Brib. & 0,16 & 0,25 & 0,19 & & & & & & & & \\
\hline Cab. & 0,17 & 0,24 & 0,19 & 0,71 & & & & & & & \\
\hline $\mathrm{Te}$ & 0,08 & 0,15 & 0,12 & 0,23 & 0,21 & & & & & & \\
\hline Bor. & 0,11 & 0,18 & 0,14 & 0,24 & 0,23 & 0,17 & & & & & \\
\hline Mov. & 0,09 & 0,14 & 0,14 & 0,27 & 0,18 & 0,21 & 0,21 & & & & \\
\hline Boc. & 0,12 & 0,21 & 0,17 & 0,25 & 0,23 & 0,18 & 0,22 & 0,30 & & & \\
\hline $\mathrm{Cu}$ & 0,10 & 0,18 & 0,15 & 0,18 & 0,16 & 0,18 & 0,21 & 0,20 & 0,20 & & \\
\hline Cág. & 0,11 & 0,21 & 0,21 & 0,24 & 0,26 & 0,18 & 0,21 & 0,21 & 0,25 & 0,23 & \\
\hline Bint. & 0,11 & 0,17 & 0,18 & 0,19 & 0,19 & 0,17 & 0,18 & 0,21 & 0,23 & $0,19 \quad 0,48$ & \\
\hline Mu. & 0,18 & 0,23 & 0,21 & 0,26 & 0,25 & 0,19 & 0,22 & 0,18 & 0,24 & $0,21 \quad 0,31$ & 0,29 \\
\hline
\end{tabular}




\section{BIBLIOGRAFIA}

1. Agüero, Arturo. El español de América y Costa 1962 Rica. San José: Librería e Imprenta Atenea.

2. Bákit, Oscar. Garavito: nuestra raíz perdida. 1981 San José: Jiménez y Tanzi.

3. Brinton, Daniel G. "The ethnic affinities of the 1897 Guetares of Costa Rica". Proceedings of the American Philosophical Society 36: 496-8.

4. Campbell, Lyle. "Middle American Languages". 1979 En: Lyle Campbell y Marianne Mithun (compiladores) The languages of Native America: Historical and Comparative Assessment, pp. 902-1000. Austin: University of Texas Press.

5. Constenla Umaña, Adolfo. "Comparative Chib1981 chan Phonology", Tesis doctoral, Universidad de Pensilvania.

1982 "La nasalización en relación con el tono y la intensidad en bribri". Revista de Filología y Lingüística de la Universidad de Costa Rica. VIII (1 y 2): 109-120.

6. Constenla Umaña, Adolfo, Enrique Margery Pe1978 ña. Bribri /. San José: Departamento de Publicaciones de la Universidad de Costa Rica.

1979 Bribri II. San José: Departamento de Publicaciones de la Universidad de Costa Rica.

7. Corominas, Joan. Tópica Hespérica, Tomo I. 1972 Madrid: Editorial Gredos, S.A.

8. Fernández, León. Documentos para la historia 1881- de Costa Rica, 10 Volúmenes. San Jo1907 sé: París, Barcelona.

1975 Historia de Costa Rica durante la Dominación Española 1502-1821. San José: Editorial Costa Rica.
9. Fernández Guardia, Ricardo. El descubrimien1975 to y la conquista. Reseña histórica de Talamanca. San José: Editorial Costa Rica.

10. Gagini, Carlos. Los aborígenes de Costa Rica. 1917 San José: Imprenta Trejos Hnos.

11. Greenberg, J.H. "The general classification of 1960 Central and South American languages". En: Men and cultures: selected papers of the $5^{\text {th }}$ International Congress of Anthropological and Ethnogical Sciences, Philadelphia, September 1965, compilado por Anthony F.C. Wallace. Filadelfia: University of Pennsylvania Press.

1966 Languages of Africa. The Hague: Mouton.

12. Jijón y Caamaño, Jacinto. El Ecuador Interan1943 dino y Occidental, Vol. 3: Las lenguas del sur de Centroamérica y el norte y centro del Oeste de Sud-América. Quito: Editorial Ecuatoriana.

13. Lázaro Carreter, Fernando. Diccionario de tér1962 minos filológicos. Madrid: Editorial Gredos S.A.

14. Lehmann, Walter. Zentral-Amerika, 2 volúme1920 nes. Berlin: Verlag Dietrich Reimer.

15. Loukotka, Cestmir. "Klassifikation der süda1944 merikanischen Sprachen". Zeitschrift für Ethnologie 74: 1-69.

1968 Classification of South American Indian Languages. Editado por Johannes Wilbert. Los Angeles: Latin American Studies Center, The University of California at Los Angeles.

16. Margery Peña, Enrique. Diccionario fraseológi1982 co bribri-español, español-bribri. San José: Editorial Universidad de Costa Rica. 
17. Mason, John Alden. "The languages of South 1950 American Indians". En: J.H. Steward (comp.) Handbook of South American Indians, 6:157-317.

18. McQuown, Norman A. "The indigenous Ian1955 guages of Latin America". American Anthropologist 57: 501-70.

19. Morínigo, Marcos A. Programa de Filología 1959 Hispánica. Buenos Aires: Editorial Nova.

20. Pérez Zeledón, Pedro. "Los aborígenes de Cos1918 ta Rica". La Información, Año X, No. 3087 , p. 2, 6 de enero de 1918. San José.

21. Rivet, Paul. "Les familles linguistiques du 1912 Nord-Ouest de I'Amérique du Sud". L'Anné Linguistique 4:117-54.

22. Schmidt, Wilhelm. Die Sprachfamilien und 1926 Sprachenkriese der Erde. Heidelberg: Winter.

23. Swadesh, Morris. "Towards greater accuracy in 1955 lexicostatistic dating". International Journal of American Linguistics. 21: 121-37.
1959 Mapas de la clasificación lingüistica de México y las Américas. Méjico: Universidad Nacional Autónoma de México.

24. Szemerényi, Oswald. "Principles of etymologi1962 cal research in the Indo-European Ianguages". Innsbrucker Beitrage zur Kulturwissenschaft 15:175-212.

25. Thomas Cyrus. "Provisional list of linguistic fa1902 milies, languages, and dialects of Mexico and Central America". American Anthropologist, N.S. 4:207-216.

26. Thomas, Cyrus y J.R. Swanton. Indian langua1911 ges of Mexico and Central America. Smithson Instit. Bur. of Am. Ethnol. Bulletin 44. Washington.

27. Tovar, Antonio. Catálogo de las lenguas de A1961 mérica del Sur. Buenos Aires: Editorial Sudamericana.

28. Vásquez de Coronado, Juan. Cartas de Rela1964 ción sobre la Conquista de Costa Rica. San José: Academia de Geografía e Historia de Costa Rica.

29. Voegelin, C.F. y F.M. Voegelin. "Languages of 1965 the world: native America, fascicle 2". Anthropological linguistics 7 (7). 